\title{
Two Simple Proofs of Winquist's Identity
}

\author{
Chutchai Nupet \\ Department of Mathematics \\ Faculty of Science, Prince of Songkla University \\ Hatyai, Songkhla 90112, Thailand \\ por075@hotmail.com \\ Sarachai Kongsiriwong \\ Department of Mathematics \\ Faculty of Science, Prince of Songkla University \\ Hatyai, Songkhla 90112, Thailand \\ sarachai.k@psu.ac.th \\ Submitted: May 31, 2010; Accepted: Aug 12, 2010; Published: Aug 24, 2010 \\ Mathematics Subject Classification: 11F20, 11F27
}

\begin{abstract}
We give two new proofs of Winquist's identity. In the first proof, we use basic properties of cube roots of unity and the Jacobi triple product identity. The latter does not use the Jacobi triple product identity.
\end{abstract}

\section{Introduction}

Winquist's identity was discovered by L. Winquist [6] in 1969. He used it to prove the congruence

$$
p(11 n+6) \equiv 0(\bmod 11)
$$

where $p(n)$ is the number of partitions of the positive integer $n$. In 1972, L. Carlitz and M. V. Subbarao [1] gave a simple proof and a generalization of Winquist's identity. In 1987, M. D. Hirschhorn [3] gave another generalization of Winquist's identity. In 1997, S.-Y. Kang [4] gave a simple proof using the Jacobi triple product identity, the quintuple product identity and two other identities from Ramanujan's notebooks. In 2003, S. Kongsiriwong and Z.-G. Liu [5] gave a simple proof using the Jacobi triple product identity and some properties of cube roots of unity. In this paper we give two new proofs of Winquist's identity: for any complex number $q$ with $|q|<1$, and any nonzero complex numbers $a$ 
and $b$,

$$
\begin{aligned}
\sum_{m=-\infty}^{\infty} \sum_{n=-\infty}^{\infty} & (-1)^{m+n} q^{\left(3 m^{2}+3 n^{2}+3 m+n\right) / 2} \\
& \left(a^{-3 m} b^{-3 n}-a^{-3 m} b^{3 n+1}-a^{-3 n+1} b^{-3 m-1}+a^{3 n+2} b^{-3 m-1}\right) \\
= & (q ; q)_{\infty}^{2}(a ; q)_{\infty}\left(a^{-1} q ; q\right)_{\infty}(b ; q)_{\infty}\left(b^{-1} q ; q\right)_{\infty} \\
& (a b ; q)_{\infty}\left(a^{-1} b^{-1} q ; q\right)_{\infty}\left(a b^{-1} ; q\right)_{\infty}\left(a^{-1} b q ; q\right)_{\infty}
\end{aligned}
$$

where $(a ; q)_{\infty}$ denotes $\prod_{n=1}^{\infty}\left(1-a q^{n-1}\right)$. In both proofs, we will use the fact about $\omega=$ $\exp (2 \pi i / 3)$ that, for any complex number $a$,

$$
(1-a)(1-a \omega)\left(1-a \omega^{2}\right)=1-a^{3}
$$

\section{First Proof}

In this section, we prove Winquist's identity by using some properties of cube roots of unity and the Jacobi triple product identity: for each pair of complex numbers $z$ and $q$ with $z \neq 0$ and $|q|<1$,

$$
\sum_{n=-\infty}^{\infty}(-1)^{n} q^{\left(n^{2}-n\right) / 2} z^{n}=(q ; q)_{\infty}(z ; q)_{\infty}\left(z^{-1} q ; q\right)_{\infty}
$$

Let $g(a, b)$ denote the right hand side of $(1.1)$. Since $h(z):=(z ; q)_{\infty}\left(z^{-1} q ; q\right)_{\infty}$ is analytic on $0<|z|<\infty$, we can write $h$ as a Laurent series

$$
h(z)=\sum_{n=-\infty}^{\infty} a_{n} z^{n}
$$

Since $g(a, b)=(q ; q)_{\infty}^{2} h(a) h(b) h(a b) h\left(a b^{-1}\right)$, we can write $g$ as a double Laurent series

$$
g(a, b)=\sum_{m=-\infty}^{\infty} \sum_{n=-\infty}^{\infty} c_{m, n} a^{m} b^{n}=\sum_{m=-\infty}^{\infty} c_{m} a^{m}
$$

where $c_{m}=\sum_{n=-\infty}^{\infty} c_{m, n} b^{n}$. From the definition of $g$, we find that $g(a, b)=-a^{3} g(a q, b)$ and $g(a, b)=-a^{3} g\left(a^{-1}, b\right)$. Thus, from (2.1), we have, for all integers $m, c_{m}=-q^{m-3} c_{m-3}$ and $c_{m}=-c_{-m+3}$. The first equation implies that, for each $m$,

$$
\begin{aligned}
c_{3 m} & =(-1)^{m} q^{\left(3 m^{2}-3 m\right) / 2} c_{0}, \\
c_{3 m+1} & =(-1)^{m} q^{\left(3 m^{2}-m\right) / 2} c_{1}, \\
c_{3 m+2} & =(-1)^{m} q^{\left(3 m^{2}+m\right) / 2} c_{2},
\end{aligned}
$$


whereas the second equation implies that $c_{1}=-c_{2}$. By putting all these together, we have

$$
\begin{aligned}
g(a, b)= & c_{0} \sum_{m=-\infty}^{\infty}(-1)^{m} q^{\left(3 m^{2}-3 m\right) / 2} a^{3 m}+c_{1} \sum_{m=-\infty}^{\infty}(-1)^{m} q^{\left(3 m^{2}-m\right) / 2} a^{3 m+1} \\
& -c_{1} \sum_{m=-\infty}^{\infty}(-1)^{m} q^{\left(3 m^{2}+m\right) / 2} a^{3 m+2} .
\end{aligned}
$$

By putting $a=\omega$ in (2.2), we have

$$
g(\omega, b)=c_{0} \sum_{m=-\infty}^{\infty}(-1)^{m} q^{\left(3 m^{2}-3 m\right) / 2}+c_{1}\left(\omega-\omega^{2}\right) \sum_{m=-\infty}^{\infty}(-1)^{m} q^{\left(3 m^{2}-m\right) / 2} .
$$

Note that, by using the Jacobi triple product identity, we have $\sum_{m=-\infty}^{\infty}(-1)^{m} q^{\left(3 m^{2}-3 m\right) / 2}=0$. Then

$$
g(\omega, b)=c_{1}\left(\omega-\omega^{2}\right) \sum_{m=-\infty}^{\infty}(-1)^{m} q^{\left(3 m^{2}-m\right) / 2} .
$$

From the definition of $g$ and (1.2),

$$
g(\omega, b)=-b^{-1}\left(\omega-\omega^{2}\right)(q ; q)_{\infty}\left(q^{3} ; q^{3}\right)_{\infty}\left(b^{3} ; q^{3}\right)_{\infty}\left(b^{-3} q^{3} ; q^{3}\right)_{\infty} .
$$

From $(2.3),(2.4)$, and the Jacobi triple product identity, we obtain

$$
c_{1}=-b^{-1} \sum_{m=-\infty}^{\infty}(-1)^{m} q^{\left(3 m^{2}-3 m\right) / 2} b^{3 m} .
$$

By letting $a=b$ in (2.2), we have

$$
\begin{aligned}
0=g(b, b)= & c_{0} \sum_{m=-\infty}^{\infty}(-1)^{m} q^{\left(3 m^{2}-3 m\right) / 2} b^{3 m}+c_{1} \sum_{m=-\infty}^{\infty}(-1)^{m} q^{\left(3 m^{2}-m\right) / 2} b^{3 m+1} \\
& -c_{1} \sum_{m=-\infty}^{\infty}(-1)^{m} q^{\left(3 m^{2}+m\right) / 2} b^{3 m+2} .
\end{aligned}
$$

Using (2.5), we have

$$
c_{0}=\sum_{m=-\infty}^{\infty}(-1)^{m} q^{\left(3 m^{2}-m\right) / 2} b^{3 m}-\sum_{m=-\infty}^{\infty}(-1)^{m} q^{\left(3 m^{2}+m\right) / 2} b^{3 m+1} .
$$

Substituting $c_{0}$ and $c_{1}$ in (2.2), we obtain Winquist's identity. 


\section{Second Proof}

In this section, we prove Winquist's identity with no use of the Jacobi triple product identity. First, we let $g(a, b, q)$ denote the right hand side of (1.1). From the first proof, we write

$$
g(a, b, q)=\sum_{m=-\infty}^{\infty} \sum_{n=-\infty}^{\infty} c_{m, n}(q) a^{m} b^{n} .
$$

From Kongsiriwong and Liu's proof of Winquist's identity [5], we have

$$
\begin{aligned}
g(a, b, q)=\sum_{m=-\infty}^{\infty} \sum_{n=-\infty}^{\infty}\{ & (-1)^{m+n} q^{\left(3 m^{2}-3 m+3 n^{2}-n\right) / 2}\left(1-b q^{n}\right) a^{3 m} b^{3 n} c_{0,0}(q) \\
& \left.+(-1)^{m+n} q^{\left(3 m^{2}-m+3 n^{2}-3 n\right) / 2}\left(1-a q^{m}\right) a^{3 m+1} b^{3 n-1} c_{1,-1}(q)\right\} .
\end{aligned}
$$

Setting $a=b=q^{1 / 2}$, we obtain

$$
\begin{aligned}
0=\sum_{m=-\infty}^{\infty} \sum_{n=-\infty}^{\infty}\{ & (-1)^{m+n} q^{\left(3 m^{2}+3 n^{2}+2 n\right) / 2}\left(1-q^{n+1 / 2}\right) c_{0,0}(q) \\
& \left.+(-1)^{m+n} q^{\left(3 m^{2}+2 m+3 n^{2}\right) / 2}\left(1-q^{m+1 / 2}\right) c_{1,-1}(q)\right\} .
\end{aligned}
$$

It follows that $c_{0,0}(q)=-c_{1,-1}(q)$. Thus we have

$$
\begin{aligned}
g(a, b, q)=c_{0,0}(q) \sum_{m=-\infty}^{\infty} & \sum_{n=-\infty}^{\infty}(-1)^{m+n} q^{\left(3 m^{2}-3 m+3 n^{2}-n\right) / 2} \\
& \left\{a^{3 m} b^{3 n}-q^{n} a^{3 m} b^{3 n+1}-a^{3 n+1} b^{3 m-1}+q^{n} a^{3 n+2} b^{3 m-1}\right\} .
\end{aligned}
$$

Next, we show that $c_{0,0}(q)=1$; this part of the proof is similar to Kongsiriwong and Liu's proof of the Jacobi triple product identity [5] and Chan's proof of the quintuple product identity [2]. By putting $a=\omega$ and $b=-\omega$ in (3.1), we have

$$
\begin{aligned}
g(\omega,-\omega, q)=c_{0,0}(q) \sum_{m=-\infty}^{\infty} & \sum_{n=-\infty}^{\infty}(-1)^{m+n} q^{\left(3 m^{2}-3 m+3 n^{2}-n\right) / 2} \\
& \left\{(-1)^{n}+(-1)^{n} q^{n} \omega+(-1)^{m}-(-1)^{m} q^{n} \omega\right\} .
\end{aligned}
$$

Since

$$
(-1)^{n}+(-1)^{n} q^{n} \omega+(-1)^{m}-(-1)^{m} q^{n} \omega= \begin{cases}2 & \text { if } m \text { and } n \text { are even, } \\ -2 q^{n} \omega & \text { if } m \text { is even and } n \text { is odd } \\ 2 q^{n} \omega & \text { if } m \text { is odd and } n \text { is even } \\ -2 & \text { if } m \text { and } n \text { are odd }\end{cases}
$$


we obtain

$$
g(\omega,-\omega, q)=2(1-\omega) c_{0,0}(q)\left\{\sum_{k=-\infty}^{\infty} \sum_{l=-\infty}^{\infty} q^{6 k^{2}-3 k+6 l^{2}-l}-\sum_{k=-\infty}^{\infty} \sum_{l=-\infty}^{\infty} q^{6 k^{2}-3 k+6 l^{2}-5 l+1}\right\} .
$$

By the definition of $g$ and (1.2), we have

$$
g(\omega,-\omega, q)=2(1-\omega)(q ; q)_{\infty}\left(-q^{3} ; q^{3}\right)_{\infty}\left(q^{6} ; q^{6}\right)_{\infty} .
$$

By (3.2) and (3.3), we have

$$
\begin{aligned}
(q ; q)_{\infty}( & \left.-q^{3} ; q^{3}\right)_{\infty}\left(q^{6} ; q^{6}\right)_{\infty} \\
& =c_{0,0}(q)\left\{\sum_{k=-\infty}^{\infty} \sum_{l=-\infty}^{\infty} q^{6 k^{2}-3 k+6 l^{2}-l}-\sum_{k=-\infty}^{\infty} \sum_{l=-\infty}^{\infty} q^{6 k^{2}-3 k+6 l^{2}-5 l+1}\right\} .
\end{aligned}
$$

Next, we evaluate $g\left(-\omega^{2} q,-\omega q, q^{4}\right)$. By (3.1), we have

$$
\begin{aligned}
g\left(-\omega^{2} q,-\omega q, q^{4}\right)=(1-\omega) c_{0,0}\left(q^{4}\right)\{ & \sum_{m=-\infty}^{\infty} \sum_{n=-\infty}^{\infty} q^{6 m^{2}-3 m+6 n^{2}-n} \\
& \left.-\sum_{m=-\infty}^{\infty} \sum_{n=-\infty}^{\infty} q^{6 m^{2}-3 m+6 n^{2}-5 n+1}\right\} .
\end{aligned}
$$

Again, we evaluate $g\left(-\omega^{2} q,-\omega q, q^{4}\right)$ as an infinite product:

$$
\begin{aligned}
g\left(-\omega^{2} q,-\omega q, q^{4}\right)= & \left(q^{4} ; q^{4}\right)_{\infty}^{2}\left(-\omega^{2} q ; q^{4}\right)_{\infty}\left(-\omega q^{3} ; q^{4}\right)_{\infty}\left(-\omega q ; q^{4}\right)_{\infty}\left(-\omega^{2} q^{3} ; q^{4}\right)_{\infty} \\
& \left(q^{2} ; q^{4}\right)_{\infty}\left(q^{2} ; q^{4}\right)_{\infty}\left(\omega ; q^{4}\right)_{\infty}\left(\omega^{2} q^{4} ; q^{4}\right)_{\infty} \\
= & \frac{(1-\omega)\left(q^{12} ; q^{12}\right)_{\infty}\left(q^{4} ; q^{4}\right)_{\infty}\left(q^{2} ; q^{4}\right)_{\infty}^{2}\left(-q^{3} ; q^{12}\right)_{\infty}\left(-q^{9} ; q^{12}\right)_{\infty}}{\left(-q ; q^{4}\right)_{\infty}\left(-q^{3} ; q^{4}\right)_{\infty}} \\
= & \frac{(1-\omega)\left(q^{12} ; q^{12}\right)_{\infty}\left(q^{2} ; q^{2}\right)_{\infty}\left(q^{2} ; q^{4}\right)_{\infty}\left(-q^{3} ; q^{6}\right)_{\infty}}{\left(-q ; q^{2}\right)_{\infty}} \\
= & \frac{(1-\omega)\left(q^{6} ; q^{6}\right)_{\infty}\left(-q^{6} ; q^{6}\right)_{\infty}(q ; q)_{\infty}(-q ; q)_{\infty}\left(q^{2} ; q^{4}\right)_{\infty}\left(-q^{3} ; q^{6}\right)_{\infty}}{\left(-q ; q^{2}\right)_{\infty}} \\
= & (1-\omega)\left(q^{6} ; q^{6}\right)_{\infty}\left(-q^{3} ; q^{3}\right)_{\infty}(q ; q)_{\infty} .
\end{aligned}
$$

Substituting (3.6) in (3.5), we obtain

$$
\begin{aligned}
(q ; q)_{\infty}\left(-q^{3} ; q^{3}\right)_{\infty}\left(q^{6} ; q^{6}\right)_{\infty}=c_{0,0}\left(q^{4}\right)\{ & \sum_{m=-\infty}^{\infty} \sum_{n=-\infty}^{\infty} q^{6 m^{2}-3 m+6 n^{2}-n} \\
& \left.-\sum_{m=-\infty}^{\infty} \sum_{n=-\infty}^{\infty} q^{6 m^{2}-3 m+6 n^{2}-5 n+1}\right\} .
\end{aligned}
$$

Comparing (3.4) with (3.7), we see that $c_{0,0}(q)=c_{0,0}\left(q^{4}\right)$. It follows that

$$
c_{0,0}(q)=c_{0,0}\left(q^{4}\right)=c_{0,0}\left(q^{16}\right)=\ldots=c_{0,0}\left(q^{4^{k}}\right)=\ldots=c_{0,0}(0)=1 .
$$

Hence we have proved Winquist's identity. 


\section{References}

[1] L. Carlitz and M. V. Subbarao, A simple proof of the quintuple product identity, Proc. Amer. Math. Soc. 32(1972), 42-44.

[2] H.-C. Chan, Another simple proof of the quintuple product identity, Internat. J. Math. Math. Sci. 15(2005), 2511-2515.

[3] M. D. Hirschhorn, A generalisation of Winquist's identity and a conjecture of Ramanujan, J. Indian Math. Soc. 51(1987), 49-55.

[4] S.-Y. Kang, A new proof of Winquist's identity, J. Combin. Theory 78(1997), 313-318.

[5] S. Kongsiriwong and Z.-G. Liu, Uniform proofs of $q$-series-product identities, Results in Math. 44(2003), 312-339.

[6] L. Winquist, An elementary proof of $p(n) \equiv 0(\bmod 11)$, J. Combin. Theory 6(1969), $56-59$. 